\title{
CHAOTIC SYNCHRONIZATION OF PIECEWISE LINEAR MAPS
}

\author{
ACILINA CANECO \\ CIMA-UE and Mathematics Unit, DEETC, \\ Instituto Superior de Engenharia de Lisboa, \\ Rua Conselheiro Emídio Navarro, 1, 1949-014 Lisboa, Portugal \\ E-mail: acilina@deetc.isel.ipl.pt \\ CLARA GRÁCIO \\ Department of Mathematics, Universidade de Évora and CIMA-UE, \\ Rua Romão Ramalho, 59, 7000-671 Évora,Portugal \\ E-mail:mgracio@uevora.pt \\ J. LEONEL ROCHA \\ Mathematics Unit, DEQ, Instituto Superior de Engenharia de Lisboa, \\ Rua Conselheiro Emídio Navarro, 1, 1949-014 Lisboa, Portugal \\ E-mail: jrocha@deq.isel.ipl.pt
}

\begin{abstract}
We derive a threshold value for the coupling strength in terms of the topological entropy, to achieve synchronization of two coupled piecewise linear maps, for the unidirectional and for the bidirectional coupling. We prove a result that relates the synchronizability of two m-modal maps with the synchronizability of two conjugated piecewise linear maps. An application to the bidirectional coupling of two identical chaotic Duffing equations is given.
\end{abstract}

\section{Introduction}

Synchronization is a process wherein two or more systems starting from slightly different initial conditions would evolve in time, with completely different behaviour, but after some time they adjust a given property of their motion to a common behaviour, due to coupling or forcing. Various types of synchronization have been studied. This includes complete synchronization (CS), phase synchronization (PS), lag synchronization (LS) generalized synchronization (GS), anticipated synchronization (AS), and others $\left[{ }^{2}\right]$. The coupled systems might be identical or different, the coupling might be unidirectional, (master-slave or drive-response), or bidirectional (mutual coupling) and the driving force might be deterministic or stochastic.

In [3], A. Kenfack studied the linear stability of the coupled double-well Duffing oscillators projected on a Poincaré section. In $\left[{ }^{4}\right]$, Kyprianidis et al. observed numerically the synchronization of two identical single-well Duffing oscillators.

In this work we investigate the unidirectional and bidirectional synchronization 
of two identical $m+1$ piecewise linear maps and obtain, analytically, the value of the coupling parameter for which the complete synchronization is achieved. Then, we study the relationship between the synchronization of two coupled identical $m$-modal maps and the synchronization of the corresponding conjugated piecewise linear maps. Next, we verify numerically the chaotic synchronization of two identical bidirectionally coupled double-well Duffing oscillators.

\section{Main results}

Consider a discrete dynamical system $u_{n+1}=f\left(u_{n}\right)$, where $u=\left(u_{1}, u_{2}, \ldots, u_{m}\right)$ is an $m$-dimensional state vector with $f$ defining a vector field $f: \mathbb{R}^{m} \rightarrow \mathbb{R}^{m}$. The coupling of two such identical maps $x_{n+1}=f\left(x_{n}\right)$ and $y_{n+1}=f\left(y_{n}\right)$ defines another discrete dynamical system $\varphi: \mathbb{N}_{0} \times \mathbb{R}^{2 m} \rightarrow \mathbb{R}^{2 m}$, i.e., $\varphi(0, x, y)=(x, y)$, $\forall(x, y) \in \mathbb{R}^{2 m}$ and $\varphi(t+s, x, y)=\varphi(t, \varphi(s, x, y)), \forall(x, y) \in \mathbb{R}^{2 m}, \forall(t, s) \in \mathbb{N}_{0}^{2}$.

Denoting by $k$ the coupling parameter, if we consider an unidirectional coupling

$$
\left\{\begin{array}{l}
x_{n+1}=f\left(x_{n}\right) \\
y_{n+1}=f\left(y_{n}\right)+k\left[f\left(x_{n}\right)-f\left(y_{n}\right)\right]
\end{array},\right.
$$

then $\varphi(n, x, y)=\left(f\left(x_{n}\right), f\left(y_{n}\right)+k\left[f\left(x_{n}\right)-f\left(y_{n}\right)\right]\right)$. If the coupling is bidirectional

$$
\left\{\begin{array}{l}
x_{n+1}=f\left(x_{n}\right)-k\left[f\left(x_{n}\right)-f\left(y_{n}\right)\right] \\
y_{n+1}=f\left(y_{n}\right)+k\left[f\left(x_{n}\right)-f\left(y_{n}\right)\right]
\end{array}\right.
$$

then $\varphi(n, x, y)=\left(f\left(x_{n}\right)+k\left[f\left(y_{n}\right)-f\left(x_{n}\right)\right], f\left(y_{n}\right)+k\left[f\left(x_{n}\right)-f\left(y_{n}\right)\right]\right)$.

To be able to say if the two systems are synchronized we must look to the difference $z_{n}=y_{n}-x_{n}$ and see if this difference converges to zero, as $n \rightarrow \infty$. If the coupling is unidirectional then

$$
z_{n+1}=(1-k)\left[f\left(y_{n}\right)-f\left(x_{n}\right)\right] .
$$

If the coupling is bidirectional then

$$
z_{n+1}=(1-2 k)\left[f\left(y_{n}\right)-f\left(x_{n}\right)\right] .
$$

These two systems are said in complete synchronization if there is an identity between the trajectories of the two systems. In $\left[{ }^{7}\right]$ and $\left[{ }^{8}\right]$ it is establish that this kind of synchronization can be achieved provided that all the Lyapunov exponents are negative.

\subsection{Synchronization of piecewise linear maps}

Let $I=[a, b] \subseteq \mathbb{R}$ be a compact interval. By definition, a continuous map $f: I \rightarrow I$ which is piecewise monotone, i.e., there exist points $a=c_{0}<c_{1}<\cdots c_{m}<$ $c_{m+1}=b$ at which $f$ has a local extremum and $f$ is strictly monotone in each of the subintervals $I_{0}=\left[c_{0}, c_{1}\right], \ldots, I_{m}=\left[c_{m}, c_{m+1}\right]$, is called a $m$-modal map. As a particular case, if $f$ is linear in each subinterval $I_{0}, \ldots, I_{m}$, then $f$ is called a $m+1$ piecewise linear map. 
By theorem 7.4 from Milnor and Thurston $\left[{ }^{5}\right]$ and Parry $\left[{ }^{6}\right]$ it is known that every $m$-modal map $f: I=[a, b] \subset \mathbb{R} \rightarrow I$, with growth rate $s$ and positive topological entropy $h_{\text {top }}(f)\left(\log s=h_{\text {top }}(f)\right)$ is topologically semiconjugated to a $p+1$ piecewise linear map $T$, with $p \leqslant m$, defined on the interval $J=[0,1]$, with slope $\pm s$ everywhere and $h_{t o p}(T)=h_{t o p}(f)=\log s$, i.e., there exist a function $h$ continuous, monotone and onto, $h: I \rightarrow J$, such that $T \circ h=h \circ f$. If, in addition, $h$ is a homeomorphism, then $f$ and $T$ are said topologically conjugated.

According to the above statements, we will investigate the synchronization of two identical $p+1$ piecewise linear maps with slope $\pm s$ everywhere (Theorem 2.1.) and also the synchronization of two identical $m$-modal maps (Theorem 2.2.).

In what follows we will use the symbols $f$ and $k$ to represent, respectively, the $m$-modal map and its coupling parameter and the symbols $T$ and $c$ to represent, respectively, the $p+1$ piecewise linear map and its coupling parameter.

Let $T: J=\left[a_{1}, b_{1}\right] \subseteq \mathbb{R} \rightarrow J$, be a continuous piecewise linear map, i.e., there exist points $a_{1}=d_{0}<d_{1}<\cdots d_{p}<d_{p+1}=b_{1}$ such that $T$ is linear in each subintervals $J_{i}=\left[d_{i}, d_{i+1}\right],(i=0, \ldots, p)$, with slope $\pm s$ everywhere.

So, the unidirectional coupled system for $T$ is

$$
\left\{\begin{array}{l}
X_{n+1}=T\left(X_{n}\right) \\
Y_{n+1}=T\left(Y_{n}\right)+c\left[T\left(X_{n}\right)-T\left(Y_{n}\right)\right]
\end{array},\right.
$$

and the difference $Z_{n}=Y_{n}-X_{n}$ verifies

$$
Z_{n+1}=(1-c)\left[T\left(Y_{n}\right)-T\left(X_{n}\right)\right] .
$$

For the bidirectionally coupled system

$$
\left\{\begin{aligned}
X_{n+1} & =T\left(X_{n}\right)-c\left[T\left(X_{n}\right)-T\left(Y_{n}\right)\right] \\
Y_{n+1} & =T\left(Y_{n}\right)+c\left[T\left(X_{n}\right)-T\left(Y_{n}\right)\right]
\end{aligned}\right.
$$

the difference $Z_{n}=Y_{n}-X_{n}$ verifies

$$
Z_{n+1}=(1-2 c)\left[T\left(Y_{n}\right)-T\left(X_{n}\right)\right] .
$$

Theorem 2.1. Let $T: J \rightarrow J$, be a continuous $p+1$ piecewise linear map with slope $\pm s$ everywhere, with $s>1$. Let $c \in[0,1]$ be the coupling parameter. Then one has:

(i) The unidirectional coupled system (5) is synchronized if

$$
c>\frac{s-1}{s} \text {. }
$$

(ii) The bidirectional coupled system (7) is synchronized if

$$
\frac{s+1}{2 s}>c>\frac{s-1}{2 s} \text {. }
$$


Proof. Attending to the fact that $T$ is linear with slope $\pm s$ in each subinterval $J_{0}$, ..., $J_{p}$, then, the total variation of $T$ is

$$
V_{b_{1}}^{a_{1}}(T)=\int_{a_{1}}^{b_{1}}\left|T^{\prime}(t)\right| d t=\sum_{i=0}^{p} \int_{d_{i}}^{d_{i+1}}\left|T^{\prime}(t)\right| d t=s \sum_{i=0}^{p}\left|d_{i+1}-d_{i}\right|=s\left|b_{1}-a_{1}\right| .
$$

We have

$$
\left|T\left(Y_{n}\right)-T\left(X_{n}\right)\right|=\left|\int_{X_{n}}^{Y_{n}} T^{\prime}(t) d t\right| \leqslant \int_{X_{n}}^{Y_{n}}\left|T^{\prime}(t)\right| d t=V_{Y_{n}}^{X_{n}}(T)=s\left|Y_{n}-X_{n}\right| .
$$

Attending to (6), it follows that,

$$
\left|Z_{n+1}\right| \leqslant|(1-c) s|\left|Z_{n}\right| \text { and then }\left|Z_{q}\right| \leqslant|(1-c) s|^{q}\left|Z_{0}\right| \text {. }
$$

So, letting $q \rightarrow \infty$, we have $\lim _{q \rightarrow \infty}|(1-c) s|^{q}\left|Z_{0}\right|=0$, if $|(1-c) s|<1$. The previous arguments shows that, if $c \in[0,1]$ then the unidirectional coupled system (5) is synchronized if $c>\frac{s-1}{s}$.

On the other hand, using the same arguments as before and attending to (8), we have

$$
\left|Z_{n+1}\right| \leqslant|(1-2 c) s|\left|Z_{n}\right| \text { and then }\left|Z_{q}\right| \leqslant|(1-2 c) s|^{q}\left|Z_{0}\right| \text {. }
$$

Thus, considering $q \rightarrow \infty$, we have $\lim _{q \rightarrow \infty}|(1-2 c) s|^{q}\left|Z_{0}\right|=0$, if $|(1-2 c) s|<1$. Therefore, we may conclude that, if $c \in[0,1]$ the bidirectional coupled system (8) is synchronized if $\frac{s+1}{2 s}>c>\frac{s-1}{2 s}$.

Note that, the bidirectional synchronization occurs at half the value of the coupling parameter for the unidirectional case, as mentioned by Belykh et al $\left[^{1}\right]$.

\subsection{Conjugacy and synchronization}

In this section our question is to know the relationship between the synchronization of two coupled identical $m$-modal maps and the synchronization of the two coupled corresponding conjugated $p+1$ piecewise linear maps, with $p \leqslant m$. Consider in the interval $J$ the pseudometric defined by

$$
d(x, y)=|h(x)-h(y)| .
$$

If $h$ is only a semiconjugacy, $d$ is not a metric because one may have $d(x, y)=0$ for $x \neq y$. Nevertheless, if $h$ is a conjugacy, then the pseudometric $d$, defined above, is a metric. Two metrics $d_{1}$ and $d_{2}$ are said to be topologically equivalent if they generate the same topology. A sufficient but not necessary condition for topological equivalence is that for each $x \in I$, there exist constants $k_{1}, k_{2}>0$ such that, for every point $y \in I$,

$$
k_{1} d_{1}(x, y) \leqslant d_{2}(x, y) \leqslant k_{2} d_{1}(x, y) .
$$


Consider the pseudometric $d$ defined above, $d_{2}(x, y)=d(x, y)$ and $d_{1}(x, y)=$ $|x-y|$.

Suppose $h: I \rightarrow J$ is a bi-Lipschitz map, i.e., $\exists N, M>0$, such that,

$$
0<N|x-y| \leqslant|h(x)-h(y)| \leqslant M|x-y|, \quad \forall(x, y) \in I^{2} .
$$

If $h$ is a conjugacy and verifies (9), then the metrics $d$ and $|$.$| are equivalents.$

Consider $f: I[a, b] \subset \mathbb{R} \rightarrow I$ a $m$-modal function with positive entropy. For the unidirectional coupled system given by (1) we have the difference (3). As for the bidirectional coupled system given by (2) we have the difference (4).

As an extension of Theorem 2.1., for the synchronization of piecewise linear maps, we can establish the following result concerning the synchronization of the corresponding semiconjugated piecewise monotone maps.

Theorem 2.2. Let $f: I \rightarrow I$, be a continuous and piecewise monotone map with positive topological entropy $h_{\text {top }}=\log s$ and $h: I \rightarrow J$ a semiconjugacy between $f$ and a continuous piecewise linear map $T: J \rightarrow J$, with slope $\pm s$ everywhere. If there exist constants $N, M>0$ satisfying (9), then one has:

(i) The unidirectional coupled system (1) is synchronized if

$$
k>1-\frac{N}{M} \frac{1}{s} .
$$

(ii) The bidirectional coupled system (2) is synchronized if

$$
1+\frac{N}{M} \frac{1}{2 s}>k>1-\frac{N}{M} \frac{1}{2 s} .
$$

Proof. If $f$ is monotone in the interval $[x, y]$, then $T$ is monotone in the interval $[h(x), h(y)]$, because $h$ is monotone, so

$$
|h(f(x))-h(f(y))|=|T(h(x))-T(h(y))|=s|h(x)-h(y)| .
$$

Therefore $d(x, y)=s^{-1} d(f(x), f(y))$, if $f$ is monotone in the interval $[x, y]$. If $f$ is not monotone in the interval $[x, y]$, but there exist, points $c_{i}(i=1, \ldots, p-1)$, such that $c_{i}<c_{i+1}, c_{i} \in[x, y]$ and $f$ is monotone in each subinterval $I_{1}=\left[x=c_{0}, c_{1}\right]$, $I_{2}=\left[c_{1}, c_{2}\right], \ldots, I_{p}=\left[c_{p-1}, y=c_{p}\right]$, we have

$$
\begin{aligned}
d(x, y) & =\sum_{j=0}^{p-1} d\left(c_{j}, c_{j+1}\right) \\
& =s^{-1} \sum_{j=0}^{p-1} d\left(f\left(c_{j}\right), f\left(c_{j+1}\right)\right) \\
& =s^{-1} \sum_{j=0}^{p-1}\left|h\left(f\left(c_{j}\right)\right)-h\left(f\left(c_{j+1}\right)\right)\right| \\
& \geqslant s^{-1}|h(f(x))-h(f(y))| \\
& =s^{-1} d(f(x), f(y)) .
\end{aligned}
$$


So, we can write $d(x, y) \geqslant s^{-1} d(f(x), f(y)), \forall x, y \in I$. From (9) and for the unidirectional coupling (3) we have

$$
\begin{aligned}
d\left(y_{n+1}, x_{n+1}\right) & \leqslant M\left|y_{n+1}-x_{n+1}\right|=M|1-k|\left|f\left(y_{n}\right)-f\left(x_{n}\right)\right| \\
& \leqslant M|1-k| N^{-1} d\left(f\left(y_{n}\right), f\left(x_{n}\right)\right) \leqslant M|1-k| N^{-1} s d\left(y_{n}, x_{n}\right) .
\end{aligned}
$$

It follows that

$$
d\left(y_{n+r}, x_{n+r}\right) \leqslant M^{r}|1-k|^{r} N^{-r} s^{r} d\left(y_{n}, x_{n}\right),
$$

so $d\left(y_{n+r}, x_{n+r}\right) \underset{r \rightarrow \infty}{\rightarrow} 0$ if $\left|M(1-k) N^{-1} s\right|<1$. Then, the coupled system (1) is synchronized if

$$
k>1-\frac{N}{M} \frac{1}{s} .
$$

For the bidirectional coupling (4) and using the same arguments as before, we also have that

$$
d\left(y_{n+1}, x_{n+1}\right) \leqslant M|1-2 k| N^{-1} s d\left(y_{n}, x_{n}\right) .
$$

It follows that $d\left(y_{n+r}, x_{n+r}\right) \underset{r \rightarrow \infty}{\rightarrow} 0$ if $\left|M(1-2 k) N^{-1} s\right|<1$. Then, the coupled system (2) is synchronized if

$$
\frac{1}{2}\left(1+\frac{N}{M} \frac{1}{s}\right)>k>\frac{1}{2}\left(1-\frac{N}{M} \frac{1}{s}\right)
$$

Denote by $k^{*}$ the synchronization threshold for (1), i.e. the system of piecewise monotone functions synchronizes for $k>k^{*}$. Denote by $c^{*}$ the value such that for $c>c^{*}$ the system of piecewise linear maps (5) is synchronized. Note that

$$
N\left(1-k^{*}\right)=M\left(1-c^{*}\right) .
$$

With the assumptions we made, if the piecewise monotone coupled maps synchronizes, so do the conjugated piecewise linear coupled maps and conversely, if the piecewise linear coupled maps synchronizes, so do the conjugated piecewise monotone coupled maps. In fact, from (9) we have $d\left(y_{n}, x_{n}\right) \leqslant M\left|y_{n}-x_{n}\right|$, therefore if system (1) synchronizes for $k>k^{*}$, then system (5) synchronizes for $c>c^{*}$, because $k^{*} \geqslant c^{*}$. On the other hand, we have also from (9), $\left|y_{n}-x_{n}\right| \leqslant$ $N^{-1} d\left(y_{n}, x_{n}\right)$, therefore if the system (5) synchronizes for $c>c^{*}$, then the system (1) synchronizes for $k>k^{*}$ with $k^{*}$ verifying (10).

For the bidirectional coupling, we have

$$
1-\frac{1}{s} \leqslant 1-\frac{N}{M} \frac{1}{s} \leqslant 1+\frac{N}{M} \frac{1}{s} \leqslant 1+\frac{1}{s},
$$

so the synchronization interval for the piecewise monotone coupled maps is contained in the synchronization interval for the conjugated piecewise linear coupled maps. 


\section{Duffing oscillators's example and symbolic synchronization.}

Consider two identical bidirectionally coupled Duffing oscillators, see $\left[^{3}\right]$ and references therein

$$
\left\{\begin{array}{l}
x^{\prime \prime}(t)=x(t)-x^{3}(t)-\alpha x^{\prime}(t)+k[y(t)-x(t)]+\beta \operatorname{Cos}(w t) \\
y^{\prime \prime}(t)=y(t)-y^{3}(t)-\alpha y^{\prime}(t)-k[y(t)-x(t)]+\beta \operatorname{Cos}(w t)
\end{array}\right.
$$

where $k$ is the coupling parameter. We consider parameter values for which each uncoupled $(k=0)$ oscillator exhibits a chaotic behaviour, so if they synchronize, that will be a chaotic synchronization. We did a Poincaré section defined by $y=0$ and found in the parameter plane $(\alpha, \beta)$, a region $\mathcal{U}$ where the first return Poincaré map behaves like a unimodal map and a region $\mathcal{B}$ where the first return Poincaré map behaves like a bimodal map. We choose, for example, $w=1.18, x_{0}=0.5$, $x_{0}^{\prime}=-0.3, y_{0}=0.9, y_{0}^{\prime}=-0.2$ and $\alpha=0.4, \beta=0.3578$, for the unimodal case and $\alpha=0.5, \beta=0.719$, for the bimodal case.
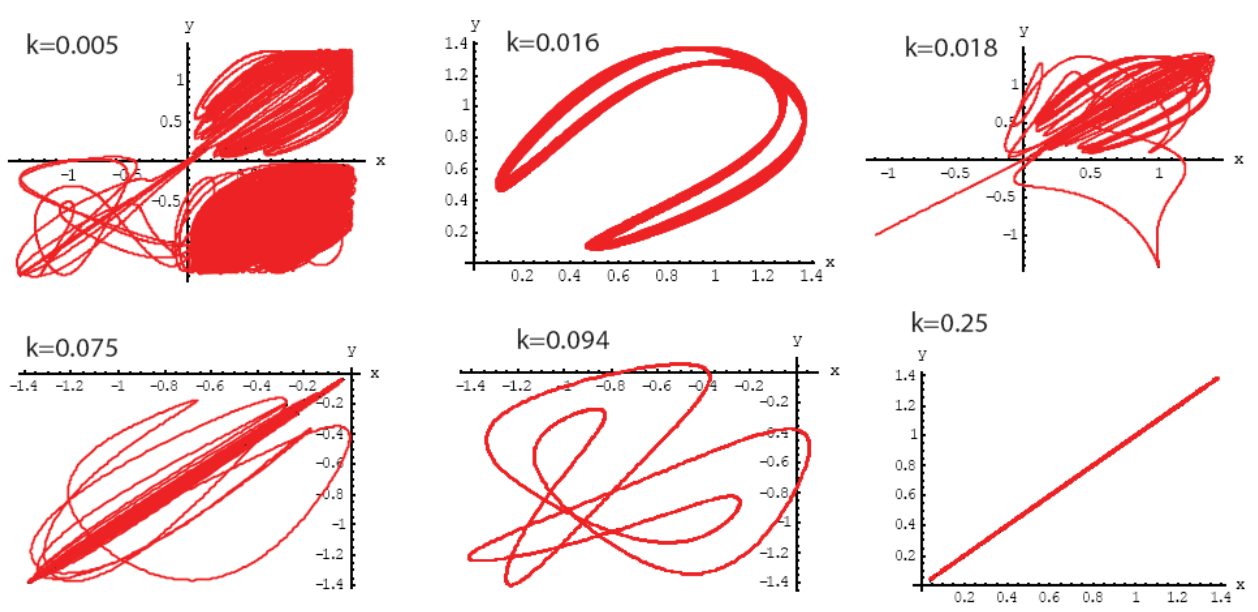

Figure 1. Evolution of $x$ versus $y$ for the bidirectional coupled Duffing oscillators, for some values of $\mathrm{k}$, in the unimodal case $(\alpha=0.4, \beta=0.3578)$.

Numerically we can see the evolution of the difference $z=y-x$ with $k$. The synchronization will occur when $x=y$. See some examples in Fig. 1 for the unimodal case. Although not shown in this figure, the graphics of the difference $y-x$ for $k$ greater then 0.122 are always a diagonal like in the picture for $k=0.25$, showing that these Poincaré unimodal maps are synchronized. For $\alpha=0.4$ and $\beta=0.3578$ we have $h=0.2406 \ldots$, then $s=1.272 \ldots$ If the coupled maps where piecewise linear maps with slope $s= \pm 1.272$, the synchronization will occurs for $c>c^{*}=$ $\frac{s-1}{2 s}=0.107$ and we see numerically that these unimodal Poincare maps for the Duffing equations synchronizes at a little greater value, $k^{*} \approx 0.122$, so these pictures confirms numerically the above theoretical results, though we cannot guarantee 
that the semiconjugation between the unimodal and the piecewise linear maps is a conjugation.

\section{Conclusions}

We obtained explicitly the value $c^{*}$ of the coupling parameter, such that for $c>c^{*}$ two piecewise linear maps, unidirectional or bidirectional coupled are synchronized. Moreover we prove that, in certain conditions, the synchronization of two $m$-modal maps is equivalent to the synchronization of the corresponding conjugated piecewise linear maps, but for different values of the coupling parameter. A numerical application to the coupling of two Duffing equations is given.

Acknowledgments: The authors are thankful to the referee for his authoritative comments and suggestions, which helped so much in improving the presentation of our results. This research has been partially supported by CIMA-UE and ISEL.

\section{References}

1. I. Belykh, M. Hasler and V. Belykh, When symmetrization guarantees synchronization in directed networks, Int. J. Bif. Chaos, 17 (10) (2007).

2. S. Boccaletti, J. Kurths, G. Osipov, D.L. Valladares and C. S. Zhou, The synchronization of chaotic systems, Physics Reports 366 (2002) 1-101.

3. A. Kenfack, Bifurcation structure of two coupled periodically driven double-well Duffing oscillators, Chaos Sol. Frac. 15 (2003) 205-218.

4. I. M. Kyprianidis, Ch. Volos, I. N. Stouboulos and J. Hadjidemetriou, Dynamics of two resistively coupled Duffing-type electrical oscillators, Int. J. Bif. Chaos, 16 (2006) 1765-1775.

5. J. Milnor and W. Thurston, On iterated maps of the interval I and II, Lect. Notes in Math, No 1342, Springer-Verlag, (1988) 465-563.

6. W. Parry, Symbolic dynamics and transformations of the unit interval, Trans. Amer. Math. Soc., 122, 368-378, (1964).

7. L. M. Pecora and T. L. Carroll, Synchronization in chaotic systems, Phys. Rev. Lett., 64, (1990) 821-824.

8. L. M. Pecora and T. L. Carroll, Driving systems with chaotic signals, Phys. Rev. A, 44, (1991) 2374-2383. 